\title{
利用组学数据建立针对四种女性癌症的基于机器 学习方法的生存预测模型
}

桑浩凯 ${ }^{1}$, 郭树理 ${ }^{2}$, 曲红 ${ }^{3^{*}}$, 赵敏 ${ }^{4^{*}}$, 曲大成 ${ }^{1^{*}}$

1. 北京理工大学计算机学院, 北京 100081 ;

2. 北京理工大学自动化学院, 北京理工大学复杂系统智能控制与决策教育重点实验室, 北京 100081 ;

3. 北京大学生命科学学院, 生物信息中心, 蛋白质与植物基因研究国家重点实验室, 北京 100871 ;

4. School of Engineering, Faculty of Science, Health, Education and Engineering, University of the Sunshine Coast, Maroochydore DC, Queensland 4558, Australia

*联系人, E-mail: quh@mail.cbi.pku.edu.cn; mzhao@usc.edu.au; qudc@bit.edu.cn

收稿日期：2019-01-14; 接受日期：2019-03-19; 网络版发表日期：2019-05-16

国家自然科学基金(批准号: 31671375)、国家重点研发计划(批准号: 2017YFC1201200, 2017YFF0207400)和澳大利亚阳光海岸大学研究启动 基金资助

摘要乳腺癌、宫颈鳞状细胞癌、子宫内膜癌、卵巢癌是女性常见的癌症. 由于癌症的恶性发展并缺乏有效的 早期诊疗手段, 这些癌症已成为当今世界女性患者的头号杀手. 为了探索高通量组学数据能否促进癌症患者的预 后, 本研究利用美国癌症基因组图谱项目中四种女性癌症的 1861 个样本的临床数据和多维组学数据(包括DNA甲 基化、mRNA表达、miRNA表达和基于芯片的蛋白表达组学数据), 建立了Cox比例风险模型和随机生存森林模 型用来回顾性地预测患者的生存率. 本研究发现, 在宫颈鳞状细胞癌中, 通过整合临床与DNA 甲基化及miRNA表 达组学数据建立的模型，生存预测能力显著高于仅使用临床数据的模型(一致性指数 c-index 中位数提高了 $8.73 \%$ 15.03\%). 本研究虽然验证了有些组学数据对特定癌症生存模型的预测能力有提升作用, 但也存在着相对于临床 数据, 组学数据对模型的预测能力无显著提升的情况. 这些结果为系统地开展基于癌症基因组学的生存预测研究 及提升临床生存分析的预测准确性提供了有用经验.

关键词癌症基因组学, 生存分析, Cox比例风险模型, 随机生存森林模型, LASSO回归

女性常发的癌症有乳腺癌、宫颈鳞状细胞癌(宫 颈癌)、子宫内膜癌和卵巢癌等. 乳腺癌是最常见的女 性恶性肿瘤，同时也是造成女性癌症患者死亡的第二 大原因. 乳腺癌在20 39岁之间最常见, 在不发达国家, 被诊断患有乳腺癌时的妇女的平均年龄比发达国家小 约10年. 有证据表明, 年轻女性乳腺癌的发病率正在增
加 $^{[1 \sim 3]}$. 宫颈癌也是世界各地主要的女性癌症类型和癌 症患者死亡的主要原因. 全世界每年有近 50 万妇女患 上这种疾病，其中大约有三分之二的患者被诊断为局 部晚期宫颈癌 ${ }^{[4,5]}$. 几乎所有的病例都是持续感染了 15 种致癌性人乳头瘤病毒(human papillomavirus, HPV) 基因型之一. HPV的每种基因型都是独立感染，有着

引用格式: 桑浩凯, 郭树理, 曲红, 等. 利用组学数据建立针对四种女性癌症的基于机器学习方法的生存预测模型. 中国科学: 生命科学, 2019, 49: 738-748 Sang H K, Guo S L, Qu H, et al. Development of omics data based survival models for four female cancers using machine learning approaches (in Chinese). Sci Sin Vitae, 2019, 49: 738-748, doi: 10.1360/N052018-00265 
不同的致癌风险 ${ }^{[6]}$. 子宫内膜癌是发达国家女性生殖 道最常见的恶性肿瘤, 子宫内膜癌主要影响绝经后妇 女. $15 \% \sim 25 \%$ 的病例在绝经前可以被诊断 ${ }^{[7]}$. 尽管辅 助以积极的治疗, 但是晚期患者 5 年生存率仍然低于 $20 \%$. 卵巢癌是世界女性中第五大常见肿瘤 ${ }^{[7]}$, 其发病 率逐年上升, 死亡率在妇科肿瘤中名列第一. 近年来卵 巢癌的发生亦呈现年轻化的趋势 ${ }^{[8 \sim 10]}$.

使用生存分析研究时间-事件结果关系有重要的 临床和统计学意义 ${ }^{[11]}$. 通常生存分析是利用各类临床 数据预测病人一定时间后的生存率. 生存分析的主要 目标是找到能够影响特定临床情况下患者存活率的因 素. Cox比例风险模型(Cox's proportional hazards regression model)是生存分析最重要也是最常用的预测 模型, 是一种半参数模型, 该模型由英国统计学家 $\mathrm{Cox}^{[12]}$ 提出, 主要用于肿瘤和其他慢性病的预后分析, 它还可以用于群组研究中的病因探索. 其主要的优点 有采用多因素分析方法, 不需要考虑生存时间分布, 可以利用截尾数据.

随机生存森林(random survival forest, RSF)模型也 是进行生存预测分析较为常用的一个模型. RSF是一 种集成生存树的方法, 继承了随机森林抗噪声、防止 过拟合、可处理非线性相关等优点, 可用于高维数据 分析和变量篮选 ${ }^{[13]}$.

尽管临床数据是进行生存分析的基础, 但是由于 临床数据中患者相关的数据属性有限, 往往并不能全 面反映病患生存预后的情况. 大量的研究探讨了通过 加入各类基因组、转录组数据来提高临床数据的生存 预测能力. 例如, Gómez-Rueda等人 ${ }^{[14]}$ 选用三种不同算 法用于建立预测模型, 分别对乳腺癌、胶质母细胞 癌、肺鳞状细胞癌和卵巢癌患者的mRNA表达、 miRNA表达、拷贝数变异(copy number variation, $\mathrm{CNV}$ )、DNA体细胞变异数据进行整合, 得到了性能 提高的模型; Lezcano-Valverde等人 ${ }^{[15]}$ 使用机器学习的 RSF方法根据来自马德里的两个独立数据集建立并验 证了类风湿性关节炎死亡概率预测模型. Zhu等人 ${ }^{[16]}$ 进行了泛癌预后评估, 提出了基于多组学的机器学习 方法, 以系统地量化高通量基因组、表观基因组和转 录组特征对预测性能的影响. Yuan等人 ${ }^{[17]}$ 使用Cox与 RSF方法分别对肾透明细胞癌、胶质母细胞癌、卵巢 浆液性囊腺癌和肺鳞状细胞癌样本构建模型, 对表现 良好的模型进行深入分析, 以获得重要的对生存有影
响的生物分子.

本研究通过整合妇科常见的乳腺癌、宫颈癌、子 宫内膜癌、卵巢癌等四种癌症患者的临床和多维组学 数据, 分别构建了基于 $\mathrm{LASSO}+\mathrm{Cox}$ 方法(简称Cox方 法)和RSF方法的生存预测模型，并构建了基于两模型 的集成模型, 以期在充分整合临床与基因组、转录组 数据的基础上, 找到具有更好预测性能的模型.

\section{1 方法}

\section{1 数据的获取}

对于乳腺癌(BRCA)、宫颈癌(宫颈鳞状细胞癌 (CESC))、子宫内膜癌 (UCEC) 和卵巢癌 $(\mathrm{OV})$ 四种癌 症, 我们从TCGA数据门户 (https://portal.gdc.cancer. gov/repository) 分别获取了四种癌症患者的临床数据. 从firehose网站(http://firebrowse.org)分别获取了四种 癌症样本相关的DNA甲基化(methylation)、miRNA表 达、mRNA表达和反相蛋白质芯片(reverse phase protein array, RPPA)数据, 生成这些数据的平台如表 1 所示.

对于每种癌症, 我们根据患者样本获取临床数据 和每种分子数据, 以构造用于建立生存预测模型的每 种类型数据的数据集.

\section{2 用于模型建立的临床数据与分子数据的预 处理}

从firehose中获得的样本的DNA甲基化和RPPA数 据已经进行了标准化处理, 因此我们对 mRNA和 miRNA表达数据使用 $Z$-score方法进行了标准化处理. 对组学数据全部使用标准化的连续型数值表示. 如针 对乳腺癌患者的某一种miRNA, 使用Z-score标准化的 方法对该miRNA表达数据进行处理. $Z$-score公式如 式(1)所示:

$z=\frac{x-\mu}{\sigma}$,

其中 $x$ 是特征原始的数据, $\mu$ 是所有样本中该特征的均 值, 分母 $\sigma$ 为所有样本中该特征的标准差.

我们发现在组学数据中存在少量的缺失值, 因此 使用中值填补的方法进行缺失值弥补. 针对DNA甲基 化, miRNA, mRNA, RPPA数据特征列中存在的缺失 值, 用该列的中值填补. DNA甲基化、miRNA表达、 
表 1 各组学数据的来源平台

Table 1 Platforms for generating omics data

\begin{tabular}{ccc}
\hline 癌症 & 分子数据 & 平台 \\
\hline DNA methylation & miRNA & Illumina Infinium Human DNA Methylation 450K \\
乳腺癌 & mRNA & Illumina Genome Analyzer/HiSeq 2000 miRNA sequencing platform \\
& RPPA & Illumina HiSeq 2000 RNA Sequencing V2 \\
& DNA methylation & MD Anderson Reverse Phase Protein Array (RPPA) Core platform \\
\hline 宫颈癌 & miRNA & Illumina Infinium Human DNA Methylation 450K \\
& mRNA & Illumina Genome Analyzer/HiSeq 2000 miRNA sequencing platform \\
子宫内膜癌 & DNA methylation & Illumina HiSeq 2000 RNA Sequencing V2 \\
\hline & miRNA & Illumina Infinium Human DNA Methylation 450K \\
& mRNA & Illumina Genome Analyzer/HiSeq 2000 miRNA sequencing platform \\
& RPPA & Illumina HiSeq 2000 RNA Sequencing V2 \\
卵巢癌 & MD Anderson Reverse Phase Protein Array (RPPA) Core platform \\
\hline & miRNA & Illumina Infinium Human DNA Methylation 27K \\
& mRNA & Agilent $8 \times$ 15K Human miRNA-specific microarray platform \\
& RPPA & Agilent 244K Custom Gene Expression G4502A
\end{tabular}

mRNA表达、RPPA数据的特征维度信息(其中宫颈癌 只有三种分子数据)如表 2 所示. 这些分子数据经过标 准化和缺失值弥补后，用于模型的建立. 对于临床数 据中字符串类型的特征，如肿瘤分期，由于其分期存 在先后顺序，因此我们使用标签编码的方式对特征列 进行编码. 对于其中的缺失值，我们用特定的标签进 行表示.

癌症样本的临床数据包含年龄、TNM分期、肿 瘤分级、治疗手段、出生日期等，但不是每一个样本 都包含全部的特征信息，且由于分期和分级等特征常 用于评估恶性肿瘤的生物学行为和预后，因此我们综 合选取年龄、TNM分期、肿瘤分级信息作为临床数 据的特征.

\subsection{Cox和RSF模型的建立}

四种癌症下包含生存数据的临床样本数、四种组 学分子数据相关的样本数(其中宫颈癌只有三种分子 数据)及最终用于建立各种模型的样本数列于表 3 . 用 于建立模型的样本数是通过整合四种癌症组学分子数 据相关的样本数得到的, 其中乳腺癌包含 620 个样本、 宫颈癌包含 291 个样本、子宫内膜癌包含 425 个样本、 卵巢癌包含 527 个样本.
在模型建立过程中，对于每个数据集，我们使用 5 倍交叉验证方法, 然后使用Cox和RSF方法进行模型的 训练. 首先, 将样本随机分为两组数据, 分别为训练数 据和测试数据，训练数据占样本的 $80 \%$, 测试数据占 $20 \%$. 对于临床数据, 我们选取临床样本数据中的年 龄、 $\mathrm{TNM}$ 分期、肿瘤分级信息作为特征，分别使用 $\mathrm{R}$ 语言“survival”包中的coxph函数和“randomForestSRC” 包对数据进行训练, 构建仅使用临床变量的Cox和RSF 模型.

对于分子数据，由于Cox方法没有特征选择的功 能, 因此在建立Cox模型之前, 使用LASSO回归对分子 数据进行特征选取, 将特征选择的结果作为新的自变 量用于建立仅使用分子数据的Cox模型. 具体的操作 是首先调取R语言中的“glmnet”包，利用其中的lasso函 数进行特征选择, 然后使用“survival”包中的coxph函数 对数据进行训练得到Cox模型. 在对RSF模型的建立 中, 由于RSF继承了随机森林的特性, 即在模型构建过 程中会自动执行特征选择，因此我们直接将分子数据 作为自变量，使用R语言中的“randomForestSRC”包对 数据进行训练, 得到仅使用分子数据的RSF模型.

除了仅使用临床变量或者仅使用分子数据建立模 型外，我们还将患者的临床变量分别与DNA甲基化、 
表 2 四种癌症的四种组学数据维度信息

Table 2 Number of each feature related with the four types of omics data of the four cancers

\begin{tabular}{ccccc}
\hline 癌症 & Methyl & miRNA & mRNA & RPPA \\
\hline 乳腺癌 & 16476 & 1047 & 20532 & 227 \\
宫颈癌 & 16295 & 1047 & 20532 & - \\
子宫内膜癌 & 16603 & 1047 & 16384 & 209 \\
卵巢癌 & 27579 & 822 & 17815 & 209 \\
\hline
\end{tabular}

表 3 四种癌症下样本数总览

Table 3 Overview of the number of samples related to the four cancers

\begin{tabular}{|c|c|c|c|c|c|c|}
\hline 癌症 & $\begin{array}{l}\text { 包含生存数据的 } \\
\text { 临床样本数 }\end{array}$ & $\begin{array}{l}\text { 与DNA甲基化数据 } \\
\text { 相关的样本数 }\end{array}$ & $\begin{array}{l}\text { 与miRNA表达数据 } \\
\text { 相关的样本数 }\end{array}$ & $\begin{array}{l}\text { 与 } \mathrm{mRNA} \text { 表达数据 } \\
\text { 相关的样本数 }\end{array}$ & $\begin{array}{c}\text { 与 } \mathrm{RPPA} \text { 数据相关 } \\
\text { 的样本数 }\end{array}$ & $\begin{array}{c}\text { 用于建立各种模型 } \\
\text { 的样本数 }\end{array}$ \\
\hline 乳腺癌 & 1081 & 791 & 1079 & 1093 & 887 & 620 \\
\hline 宫颈癌 & 294 & 307 & 307 & 304 & - & 291 \\
\hline 子宫内膜癌 & 544 & 444 & 550 & 557 & 440 & 425 \\
\hline 卵巢癌 & 577 & 592 & 578 & 547 & 426 & 527 \\
\hline
\end{tabular}

miRNA，mRNA和RPPA数据进行整合，用于建立整合 了临床变量与分子特征的生存预测模型. 在建立 Cox 模型之前, 将临床变量构建模型的残差作为响应变量, 使用lasso函数对分子数据进行特征选择，然后将特征 选择的结果与临床变量并列作为新的自变量用于建立 整合数据下的Cox模型. 而对于RSF模型，直接将分子 数据与临床数据并列作为新的自变量用于建立RSF 模型.

在获得生存预测模型后，使用测试数据对这些模 型进行测试，并计算得到各模型的一致性指数 (c-in-

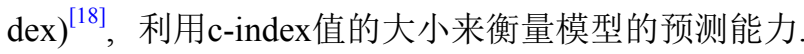
对于每个数据集, 都进行了 100 次的模型训练和测试, 并根据每次的测试计算得到一个c-index值. Wilcoxon 符号秩检验用于计算 $P$ 值以比较每种模型下 $\mathrm{c}-$ index中 位数之间的差异. c-index的取值在 $0.5 \sim 1$ 之间, c-index 等于 0.5 表示模型没有预测能力, c-index为 1 表示模型 具有完全的预测能力 ${ }^{[19]}$.

\section{4 基于Cox和RSF模型的集成模型的建立}

借鉴机器学习模型集成的思想, 对上述两种模型 赋予不同的权重, 构建集成模型:

$$
\operatorname{CoxRSF}=\alpha * \operatorname{Cox}+(1-\alpha) * \operatorname{RSF},
$$

其中的CoxRSF是集成模型, $\alpha$ 表示 0 到 1 之间的数, Cox
和RSF分别表示同一数据的Cox比例风险模型和RSF 模型， $\alpha$ 和 $(1-\alpha)$ 分别表示Cox模型和RSF模型的权重. 程序由python语言与R语言混合编写, 源代码见网络版 附录.

建立生存预测模型的实验流程如图1所示.

\section{2 结果}

\section{1 整合临床变量与组学数据构建的模型与仅使} 用临床数据或仅使用分子数据构建的模型间的比较

为了研究基因组、转录组和蛋白质组数据对生存 预测模型的预测能力的影响, 利用LASSO+Cox和RSF 方法分别建立了乳腺癌、宫颈癌、子宫内膜癌和卵巢 癌四种癌症下根据样本临床变量、分子特征、整合临 床变量与分子特征的生存预测模型。通过对每种模型 进行测试, 得到衡量模型预测能力的一致性指数 c-index的评估结果. 每种模型的c-index值的分布如图2 所示.

除了仅使用临床变量和仅使用分子数据建立模型 之外, 我们还将临床数据分别与每种组学数据进行整 合构建模型．根据各种模型的c-index值的分布，可以 看出乳腺癌、子宫内膜癌和卵巢癌中仅使用临床变量 建立的模型的预测能力均高于使用其他数据构建的模 型. 例如, 乳腺癌中临床数据的Cox和RSF模型的c-in- 


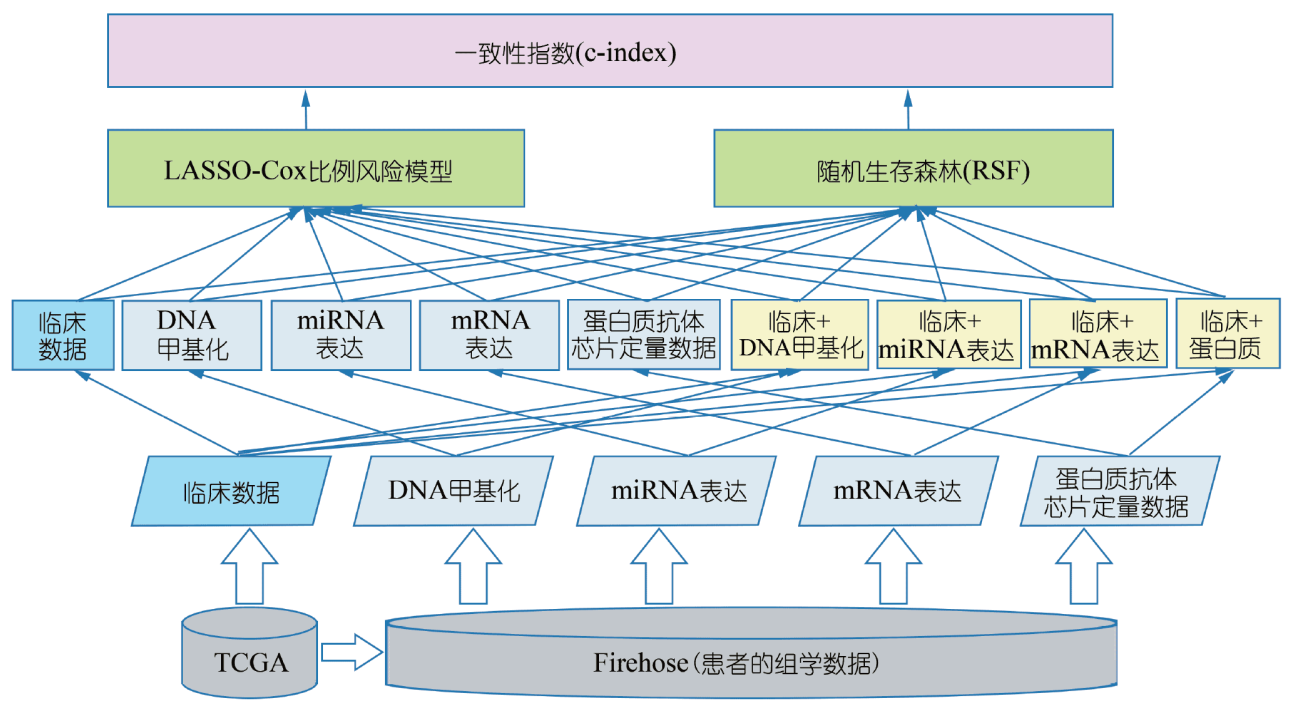

图 1 整体实验流程(网络版彩图)

Figure 1 Flow of constructing survival prediction models (color online)

dex中位数都为 0.745 ; 子宫内膜癌中临床数据的 Cox和 RSF模型的c-index中位数分别为 0.783 和 0.771 ; 卵巢癌 中临床数据的 Cox 和 RSF模型的 c-index中位数都为 0.620 . 列出的这些 $\mathrm{c}-$ index 中位数都高于其他模型的cindex中位数.

而在宫颈癌中，由整合数据建立的模型比仅使用 临床变量建立的模型具有更高的预测能力. 例如，与 仅使用临床数据构建的Cox模型相比，由DNA甲基化+ 临床数据构建的 Cox 模型的 $\mathrm{c}-$ index 中位数提高了 $8.73 \%(0.612$ 对 0.562 , WilCoxon符号秩和检验的 $P$ 值为 $\left.1.95 \times 10^{-4}\right)$; 由 miRNA+临床数据构建的Cox模型的 c-index中位数提高了 $15.03 \%(0.647$ 对 0.562 , WilCoxon 符号秩和检验的 $P$ 值为 $\left.3.536 \times 10^{-10}\right)$; 由 mRNA+临床数 据构建的Cox模型的c-index中位数提高了 $16.6 \%(0.656$ 对 0.562 , WilCoxon符号秩检验的 $P$ 值为 $4.506 \times 10^{-10}$ ). 与仅使用临床数据构建的RSF模型相比，由miRNA +临床数据构建的 RSF模型的c-index中位数增加了 $11.3 \%(0.695$ 对比 0.625 , WilCoxon符号秩检验的 $P$ 值为 $\left.1.913 \times 10^{-9}\right)$; 由 mRNA+临床数据构建的RSF模型的 c-index中位数增加了 $5.9 \%(0.662$ 对 0.625 , WilCoxon符 号秩检验的 $P$ 值为 0.001019$)$.

以上结果说明, 在不同的癌症中, 组学数据对模型 预测能力的影响不相同，宫颈癌中DNA甲基化、miRNA表达、mRNA表达数据对Cox和RSF模型的预测能
力均有一定程度的提高，而在乳腺癌、子宫内膜癌和 卵巢癌中, 组学数据没有对模型的预测能力起到提升 的效果.

与仅使用分子数据构建的模型相比，整合了临床 变量与分子组学数据的模型的性能更好. 最显著的是 子宫内膜癌, 整合数据模型的c-index中位数分别显著 增加了 $5.4 \%$ 17.8\%.

\section{2 良好预测模型中的重要生物分子识别}

在宫颈癌中，由于DNA甲基化、miRNA表达、 mRNA表达数据对模型的预测能力有一定程度的提 升, 因此我们可以进一步地根据预测模型找到对模型 预测能力有显著影响的重要生物分子. 其中临床+ miRNA数据建立的Cox模型在宫颈癌中的预测能力显 著高于单独的临床变量模型，在该模型中具有高预测 能力的重要影响因子列于表4. 通过文献调研, 发现这 些模型中的重要miRNA分子都在宫颈癌中起重要的 调节作用. 如针对 miR-142的研究发现miR-142的表达 与FIGO分期和淋巴转移呈负相关 $(P<0.001), \mathrm{miR}-142$ 通过直接靶向HMGB1，直接影响HMGB1的表达，进 而影响宫颈癌细胞的增殖和侵袭能力，增强细胞调 $亡^{[20]}$; 有研究表明miR-100在宫颈癌患者样品中下调 $(P=0.019)^{[21]}$, miR-100, miR-99a的下调使得其靶标在

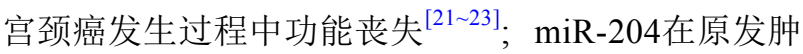



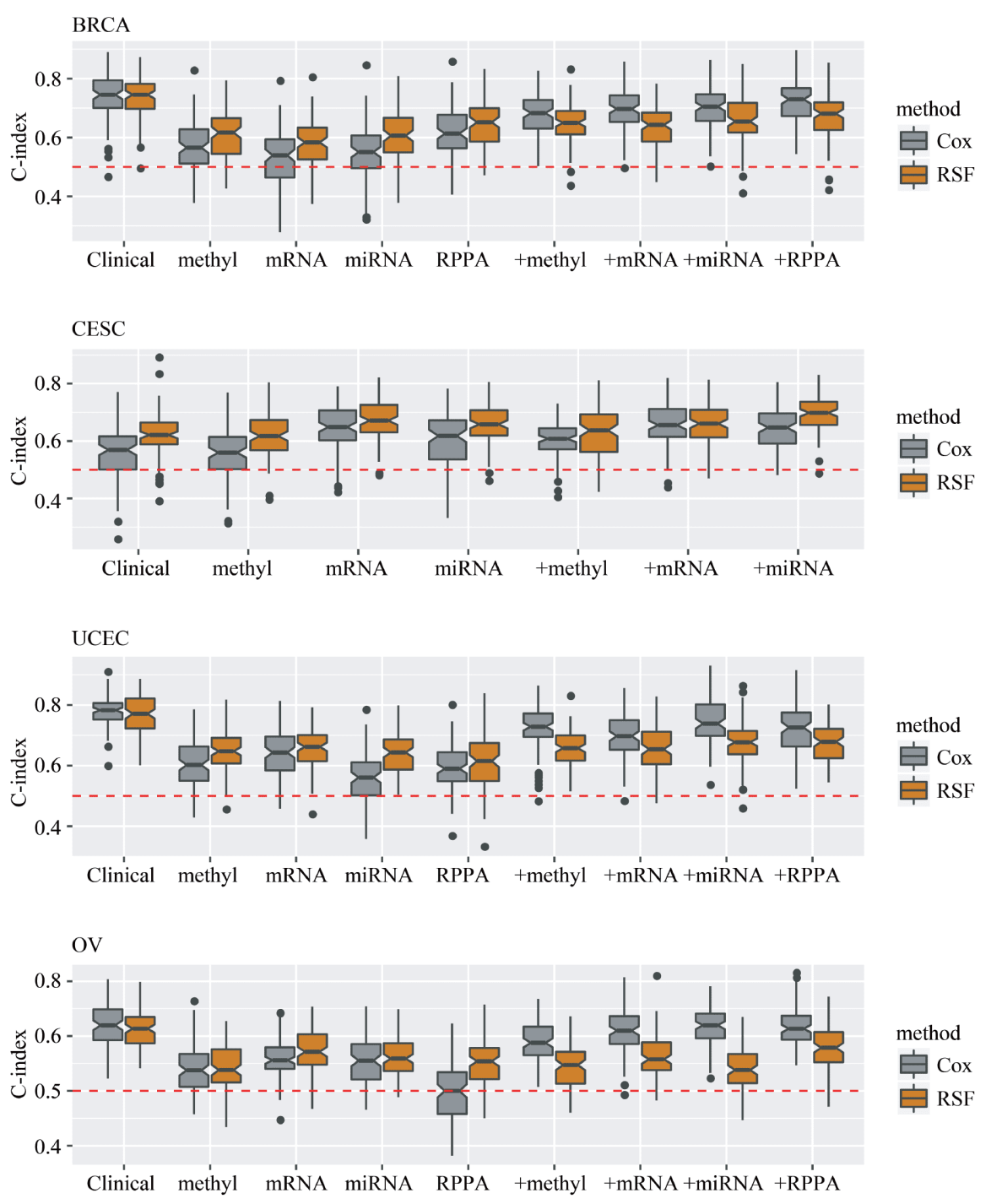

图 2 每种生存预测模型的c-index值的分布. 纵坐标表示c-index值. 图中灰色和橙色分别表示Cox和RSF方法建立的模型的 c-index分布. 横坐标上Clinical代表仅使用临床变量构建的模型; methyl, mRNA, miRNA, RPPA分别代表仅使用DNA甲基化、 mRNA表达、miRNA表达、RPPA数据构建的模型; +methyl, +mRNA, +miRNA, +RPPA分别代表通过整合临床变量与分子数 据构建的模型. 在建立每一个模型时, 将 $80 \%$ 的样本用于训练模型, 剩下的 $20 \%$ 作为测试集并用于计算c-index值

Figure 2 Distribution of c-index values for each constructed survival prediction model. The $y$-axis represents the c-index values. Gray and orange in the figures represent the c-index distribution of the models constructed by Cox and RSF methods, respectively. The "Clinical" on the $x$-axis represents the models constructed using only clinical features; "methyl", "mRNA", "miRNA" and "RPPA" represent the models constructed using only DNA methylation, mRNA expression, microRNAs expression and RPPA data, respectively; "+methyl", "+mRNA", "+microRNAs" and "+RPPA" represent the four types of models constructed by integrating clinical variables and molecular data, respectively. When constructing each model, $80 \%$ of the samples were used to train the model, and the remaining $20 \%$ were used as test sets and used to calculate the c-index values

瘤组织中的表达明显低于邻近非癌组织 $(P=0.008)$, miR-204通过靶向TCF12, 在宫颈癌的迁移和侵袭中发 挥重要作用 ${ }^{[24]}$.

另外在临床 + mRNA的Cox模型中, 具有高预测能 力的重要影响因子如表 5 所示. 研究表明外基质基因
ANGPTL4在宫颈癌细胞中表达水平显著降低 $(P<$ $0.05)^{[25]}$; 由PPP1R13L编码的蛋白质iASPP被认为不仅 抑制下游 $\mathrm{p} 53$ 的转录活性，而且还通过 $\mathrm{p} 53$ 非依赖性机 制促进癌发生 ${ }^{[26]}$; 宫颈癌中HPV感染与基因PTPN11 编码的SHP-2的表达存在显著相关性 $(P<0.05)^{[27]}$. 
表 4 宫颈癌中对基于临床+miRNA数据所建立的RSF模型的预测能力有重要影响的因子

Table 4 Important factors on the predictive performance of the RSF model constructed based on integrated clinical and miRNA data of cervical and endocervical cancer

\begin{tabular}{ccc}
\hline miRNAs & Hazard ratio & Wald's test $P$ value \\
\hline hsa-mir-142 & 0.4780 & 0.0014 \\
hsa-mir-100 & 1.2208 & 0.0567 \\
hsa-mir-147b & 1.3600 & 0.0022 \\
hsa-mir-99a & 0.6320 & 0.0465 \\
hsa-mir-204 & 1.2800 & 0.0486 \\
hsa-mir-3074 & 0.4940 & 0.0681 \\
\hline
\end{tabular}

表 5 宫颈鳞状细胞癌中基于临床+mRNA所建立模型中重要影响因子

Table 5 Important factors on the predictive performance of the model constructed based on integrated clinical and mRNA data of cervical and endocervical cancer

\begin{tabular}{ccc}
\hline mRNAs|Gene ID & Hazard ratio & Wald's test $P$ value \\
\hline ANGPTL4|51129 & 1.5531 & 0.0017 \\
PPP1R13L|10848 & 1.3423 & 0.0934 \\
PTPN11|5781 & 1.5154 & 0.0030 \\
FAM23A|653567 & 1.2057 & 0.0369 \\
ERGIC3|51614 & 1.3537 & 0.0978 \\
\hline
\end{tabular}

由此可见，这些重要影响因子之所以能够对模型 预测能力有显著影响，是因为它们与宫颈癌发生、发 展过程中的功能机制紧密相关.

\subsection{Cox 与RSF模型的集成}

为了进一步从模型角度探索是否能对模型的预测 能力进一步提升, 我们将每一个数据集的 Cox模型和 RSF模型进行集成，集成的方法请见方法1.3部分. 我 们发现集成模型的性能整体上比Cox模型和RSF模型 有明显提升. 以乳腺癌为例, 利用乳腺癌的临床 $+R P P A$ 数据训练的三种模型的c-index分布如图3所示. 发现相 对于Cox和RSF模型, 集成模型的c-index中位数都有显 著提升. 集成模型和Cox模型的 $\mathrm{c}$-index中位数分别为 0.730 和 $0.713, \mathrm{WilCox}$ on 符号秩检验的 $P$ 值为 $1.258 \times 10^{-6}$; 集成模型和 RSF模型的中位数分别为 0.730 和 0.695 , WilCoxon符号秩检验的 $P$ 值为 $4.473 \times 10^{-11}$.

在对Cox模型和RSF模型进行集成后, 集成模型的 预测能力都有显著的提升, 因此在模型层面, 可以考虑 使用模型集成的方法提升性能.

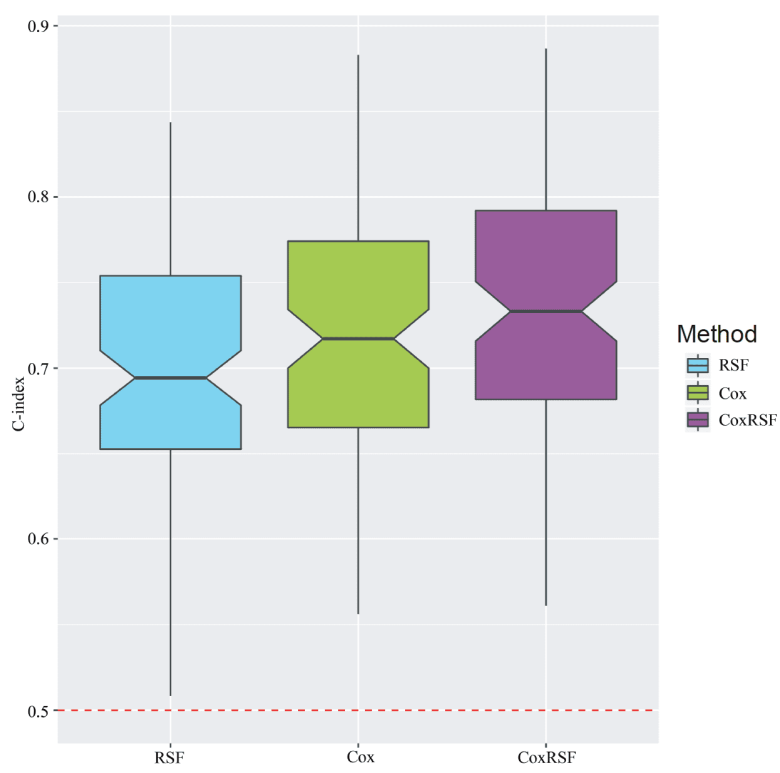

图 3 根据乳腺癌的临床+RPPA数据得到的Cox模型、RSF 模型和集成模型中的c-index值的分布. 纵坐标表示c-index 值. 图中蓝色、绿色和紫色分别表示RSF、Cox和集成模型

Figure 3 Distribution of c-index values for the three models constructed based on the integrated clinical and RPPA data of breast cancer. The c-index values are shown on the $y$-axis. Blue, green and purple in the figure represent the models constructed by RSF, Cox and integrated Cox and RSF methods, respectively 


\section{3 讨论}

在本研究中，我们系统地收集了TCGA中四种女 性癌症临床样本及相关的组学数据, 如DNA甲基化、 miRNA表达、mRNA表达、RPPA蛋白质组学数据, 分 别采用LASSO+Cox和RSF方法建立了生存预测模型 进行生存预测分析，并使用c-index指数检验模型的预 测能力. C-index是AUC(Area Under Curve)的扩展, 通 常在处理二分类数据的情况下使用 $\mathrm{AUC}$ 作为评价指 标，但它在评估和比较删失的生存数据方面具有局限 性，因为事件发生的时间通常是连续的而不是二元的， 因此我们选用c-index作为关键评价指标. 我们期望建 立良好的生存预测模型，同时找到影响模型性能的重 要生物因子, 为临床实践提供依据. 与之前由单一癌 症类型或单一数据类型驱动的研究相比, 我们系统地
评估了基于不同分子数据类型的四种癌症患者的生存 预测模型，并且提取了在性能良好模型中潜在的重要 生物因子进行功能分析，证明由模型中提取的这些潜 在重要因子确实与相关的癌症有关，这些重要因子的 发现有助于临床应用研究.

通过建立女性人群中常见的癌症(包括乳腺癌、 宫颈癌、子宫内膜癌和卵巢癌)的不同分子数据类型 的生存预测模型，发现乳腺癌、子宫内膜癌和卵巢癌 中，临床+分子模型通常比仅使用分子数据的模型具 有更好的预测能力，这是因为临床数据中包括了如年 龄、性别、癌症分期等信息，这些信息都是患者自身 各种指标的综合体现，是更高阶的特征. 使用这些包 含综合信息的特征建立的模型，其性能要比使用某一 种分子数据建立的模型更能很好地反映患者的情况并 具有更好的生存预测的准确性. 因此通过与临床数据
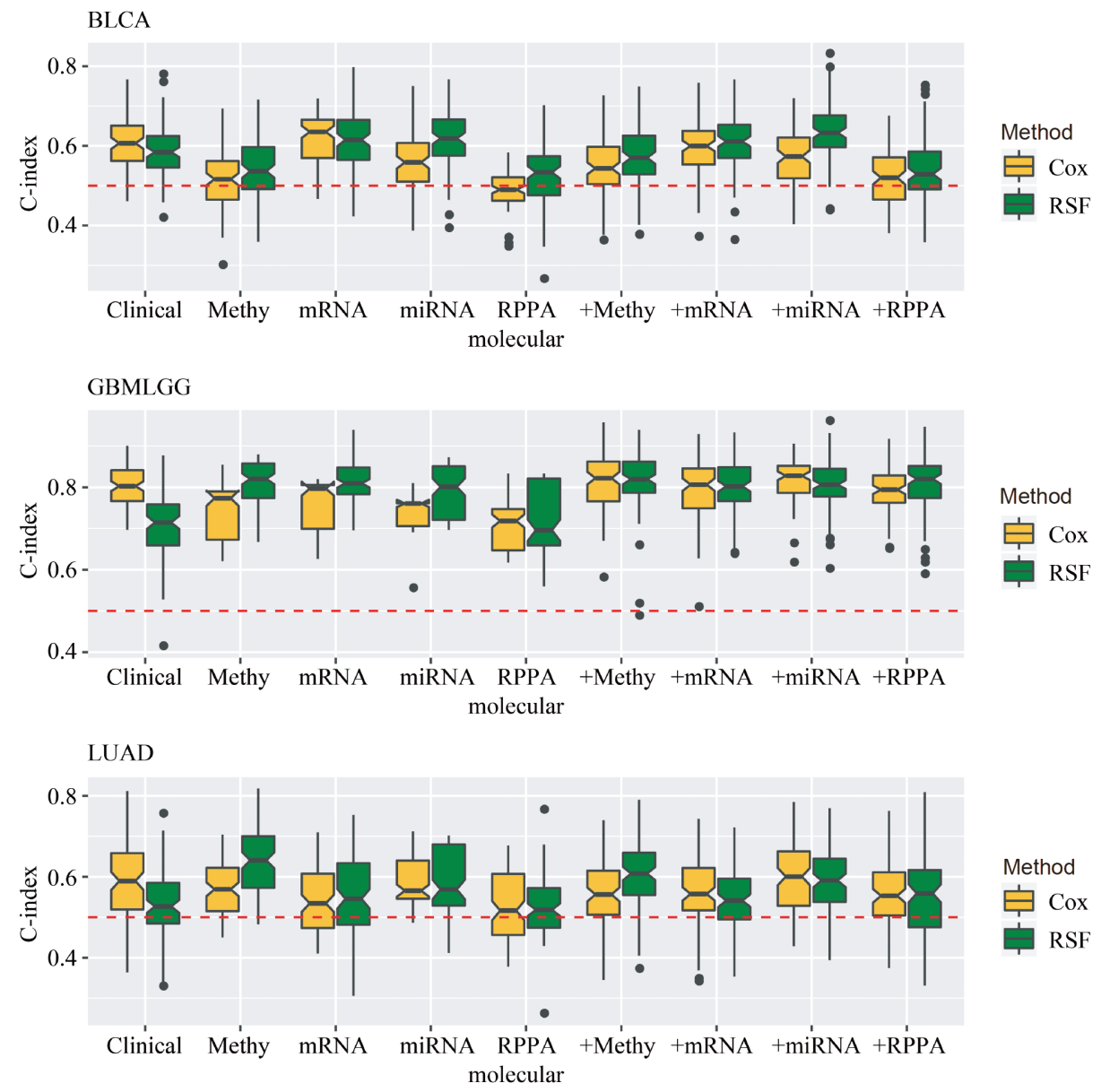

图 4 膀胱癌、胶质瘤、肺腺癌三种癌症下各生存预测模型的c-index值的分布(图中的标注与图2的意义一致)

Figure 4 Distribution of c-index values for the constructed survival prediction models of bladder cancer, glioma and lung adenocarcinoma (The labels in the figures correspond to those in Figure 2) 
的整合, 模型会产生更好的性能.

同时还发现，当仅使用分子数据构建模型的时候， RSF的性能通常高于Cox模型. 而使用整合数据构建模 型时，RSF的性能一般低于Cox模型. 这是因为RSF模 型本身继承随机森林的特点，对建立模型时的特征选 择具有随机性. 当仅使用分子数据时，不需要将分子 数据与临床数据进行相关性选择，此时RSF方法比 LASSO+Cox方法有优势. 而在使用临床和分子的整合 数据情况下，LASSO+Cox方法中是先通过LASSO回 归选取特征，那些与临床数据相关的分子数据更倾向 于被选中用于之后的模型建立，而RSF方法则会随机 选择一些特征来建立模型. 因此在整合数据情况下 LASSO+Cox比RSF方法更加有优势.

通过对那些具有更好性能的LASSO+Cox模型的 进一步分析，并从中提取了对模型预测性能有重要影 响的因子．通过文献调研，发现这些重要影响因子都 参与了癌症相关的重要生物过程, 这表明构建模型过 程中所使用的数据特征是有意义的信息. 通过鉴定这 些重要影响因子, 可能为转移性或局部疾病患者提供 个性化的治疗方案. 在本研究的最后我们将Cox和RSF 两种模型进行了集成，发现集成模型的性能有显著提 升，表明可以通过对模型的进一步处理以提高模型的 预测能力.
为了与女性癌症的生存预测模型的结论进行比 较, 进一步获取了膀胱癌(BLCA, 样本量为 325 )、胶质 瘤(GBMLGG, 样本量为418)、肺腺癌(LUAD, 样本量 为 294)的相关数据, 利用同样的方法构建了 Cox和RSF 模型, 以便于进一步考察组学数据对模型预测性能的 影响. 这三种癌症下的模型预测性能结果如图4所示. 根据图4中的c-index分布可以看到，在这三种癌症中 使用临床+分子数据的模型的性能也高于仅使用分子 数据模型的结论. 当仅使用分子数据构建模型的时候, RSF模型的性能也高于Cox模型. 这些结论与在四种女 性癌症中的模型性能比较结果是一致的. 在使用临床 +分子数据构建模型时, 膀胱癌中的RSF模型的性能高 于Cox模型, 在胶质瘤和肺腺癌中RSF的性能与Cox模 型不相上下.

尽管本研究为将生物学数据进行的生存预测结果 转化为临床应用方面提供了借鉴，但它依然存在一些 局限性. 首先, 我们单纯地采用数据挖掘的方法进行 建模，而没有充分利用由先验知识驱动获得的候选特 征; 其次, 由于大规模生物数据存在广泛的共线性, 有 效地结合多种类型的分子数据仍然存在挑战. 未来研 究方向要建立有效融合多种类型数据的预测模型; 最 后，为了进行全面的预测分析，所使用的患者样本来 源于多种处理途径, 这可能会引起异质性.

\section{参考文献}

1 Ghiasvand R, Adami H O, Harirchi I, et al. Higher incidence of premenopausal breast cancer in less developed countries; myth or truth? BMC Cancer, 2014, 14: 343

2 Merlo D F, Ceppi M, Filiberti R, et al. Breast cancer incidence trends in European women aged 20-39 years at diagnosis. Breast Cancer Res Treat, 2012, 134: 363-370

3 Peccatori F A, Lambertini M, Scarfone G, et al. Biology, staging, and treatment of breast cancer during pregnancy: Reassessing the evidences. Cancer Biol Med, 2018, 15: 6

4 Marquina G, Manzano A, Casado A. Targeted agents in cervical cancer: Beyond bevacizumab. Curr Oncol Rep, 2018, 20: 40

5 Waggoner S E. Cervical cancer. Lancet, 2003, 361: 2217-2225

6 Sales K J. Human papillomavirus and cervical cancer. In: Cancer and Inflammation Mechanisms: Chemical, Biological, and Clinical Aspects. Hoboken: John Wiley \& Sons, 2014. 165-180

7 Siegel R, Ma J, Zou Z, et al. Cancer statistics, 2014. CA A Cancer J Clin, 2014, 64: 9-29

8 Kim K, Zang R, Choi S C, et al. Current status of gynecological cancer in China. J Gynecol Oncol, 2009, 20: 72-76

9 Lee J Y, Kim E Y, Jung K W, et al. Trends in gynecologic cancer mortality in East Asian regions. J Gynecol Oncol, 2014, 25: 174-182

10 Wu Q J, Vogtmann E, Zhang W, et al. Cancer incidence among adolescents and young adults in urban Shanghai, 1973-2005. PLoS ONE, 2012, 7: e42607

11 Feng S Y. Survival analysis (II) (in Chinese). J Math Prac Theory, 1982, 3: 72-80 [冯士雍. 生存分析( II ). 数学的实践与认识, 1982, 3: 72-80]

12 Cox D R. Regression Models and Life-Tables. Breakthroughs in Statistics. New York: Springer, 1992 
13 Ishwaran H, Kogalur U B, Blackstone E H, et al. Random survival forests. Ann Appl Stat, 2008, 2: 841-860

14 Gómez-Rueda H, Martínez-Ledesma E, Martínez-Torteya A, et al. Integration and comparison of different genomic data for outcome prediction in cancer. Biodata Min, 2015, 8: 32

15 Lezcano-Valverde J M, Salazar F, León L, et al. Development and validation of a multivariate predictive model for rheumatoid arthritis mortality using a machine learning approach. Sci Rep, 2017, 7: 10189

16 Zhu B, Song N, Shen R, et al. Integrating clinical and multiple omics data for prognostic assessment across human cancers. Sci Rep, 2017, 7: 16954

17 Yuan Y, Van Allen E M, Omberg L, et al. Assessing the clinical utility of cancer genomic and proteomic data across tumor types. Nat Biotechnol, 2014, 32: 644-652

18 Harrell F E, Pryor D B, Lee K L, et al. Evaluating the yield of medical tests. JAMA, 1982, 247: 2543-2546

19 Harrell F E, Lee K L, Mark D B. Multivariable prognostic models: issues in developing models, evaluating assumptions and adequacy, and measuring and reducing errors. Statist Med, 1996, 15: 361-387

20 Jiang D, Wang H, Li Z, et al. MiR-142 inhibits the development of cervical cancer by targeting HMGB1. Oncotarget, 2017, 8: 4001

21 Jiménez-Wences H, Peralta-Zaragoza O, Fernández-Tilapa G. Human papilloma virus, DNA methylation and microRNA expression in cervical cancer (Review). Oncol Rep, 2014, 31: 2467-2476

22 Chen D, Chen Z, Jin Y, et al. MicroRNA-99 family members suppress Homeobox A1 expression in epithelial cells. PLoS ONE, 2013, 8: e80625

23 Granados-López A J, Ruiz-Carrillo J L, Servín-González L S, et al. Use of mature miRNA strand selection in miRNAs families in cervical cancer development. Inter J Mol Sci, 2017, 18: 407

24 Shu L, Zhang Z, Cai Y. MicroRNA-204 inhibits cell migration and invasion in human cervical cancer by regulating transcription factor 12 . Oncol Lett, 2018, 15: 161-166

25 Zhang R, Lu H, Lyu Y Y, et al. E6/E7-P53-POU2F1-CTHRC1 axis promotes cervical cancer metastasis and activates Wnt/PCP pathway. Sci Rep, 2017, 7: 44744

26 Dong P, Ihira K, Hamada J, et al. Reactivating p53 functions by suppressing its novel inhibitor iASPP: A potential therapeutic opportunity in p53 wild-type tumors. Oncotarget, 2015, 6: 19968

27 Tao X, Shen J, Pan W, et al. Significance of SHP-1 and SHP-2 expression in human papillomavirus infected Condyloma acuminatum and cervical cancer. Pathol Oncol Res, 2008, 14: 365-371 


\title{
Development of omics data based survival models for four female cancers using machine learning approaches
}

\author{
SANG HaoKai ${ }^{1}$, GUO ShuLi ${ }^{2}$, QU Hong ${ }^{3}$, ZHAO Min ${ }^{4} \&$ QU DaCheng ${ }^{1}$ \\ 1 School of Computer Science \& Technology, Beijing Institute of Technology, Beijing 100081, China; \\ 2 State Key Laboratory of Intelligent Control and Decision of Complex Systems, School of Automatic Control, Beijing Institute of Technology, \\ Beijing 100081, China; \\ 3 Center for Bioinformatics, State Key Laboratory of Protein and Plant Gene Research, College of Life Sciences, Peking University, \\ Beijing 100871, China \\ 4 School of Engineering, Faculty of Science, Health, Education and Engineering, University of the Sunshine Coast, Maroochydore DC, \\ Queensland 4558, Australia
}

Breast cancer, cervical and endocervical cancer, endometrial cancer and ovarian cancer are common cancers in women. Due to the malignant development of cancer and the lack of effective early diagnosis and prognosis monitor, these cancers are the top diseases causing death among female patients. To explore whether high-throughput omics data can contribute to the prognosis of cancer patients, this study used clinical data and multidimensional omics data (including DNA methylation, mRNA expression, miRNA expression and chip-based protein expression data) of 1861 samples of four female cancers in the Cancer Genome Atlas project to construct Cox proportional hazards models and random survival forest models for retrospective prediction of patient survival. Our systematic integration found that DNA methylation and miRNA expression data could significantly improve the survival predictability in patients with cervical and endometrial cancers compared with clinical data alone (the prediction efficiency increased by $8.73 \%-15.03 \%$ ). Although some omics data contribute to the performance improvement of survival prediction models for specific cancer patients, it does not improve the predictive performance of models in other cancers. In conclusion, our study provide the insights into the omics-based survival predictions, which may have important contributions to improving the predictive accuracy of clinical survival analysis.

cancer genomics, survival analysis, Cox proportional hazards model, random survival forest, LASSO regression

doi: $10.1360 / \mathrm{N} 052018-00265$ 